\section{Large left posterior fossa meningioma presenting with quadriplegia in a woman with history of carbidopa-levodopa resistant parkinsonism}

\author{
Antonio Jose Reyes, ${ }^{1}$ Kanterpersad \\ Ramcharan, 2 Maria Alvarez, ${ }^{3}$ \\ Wesley Greaves, ${ }^{4}$ Fidel Rampersad ${ }^{5}$ \\ 1Neurology Unit, San Fernando \\ Teaching Hospital; 2Department of \\ Medicine and 5 Radiology Unit, Surgi- \\ Med Clinic; 3Department of Neurosu- \\ rgery, San Fernando Teaching Hospital; \\ 4Department of Pathology and \\ Laboratory, San Fernando Teaching \\ Hospital, Trinidad and Tobago, West \\ Indies
}

\begin{abstract}
A 56-year-old Afro-Trinidadian woman with a history of a carbidopa-levodopa resistance Parkinsonian-like syndrome for 2 years, presented with acute onset spastic quadriplegia and decreased responsiveness. Diagnosis involved clinical and MR-imaging correlation consistent with a large left posterior fossa meningioma. Surgical removal of the tumor led to complete reversibility of the neurological manifestations associated with cerebellar tonsillar herniation beyond the foramen magnum and mass effect on the brainstem, cerebellum and midbrain regions. Pathological findings were typical of a meningioma. This case demonstrates the association of a large left posterior fossa meningioma and carbidopalevodopa resistant parkinsonism in an Afro-Trinidadian woman who presented with acute onset acute quadriplegia and decreased responsiveness. This case reminds clinicians that patients with dopa unresponsiveness and/or acute neurological deficit or deterioration should be worked up for other possible causes and adds to the literature on the association of parkinsonism and intracranial space occupying lesions.
\end{abstract}

\section{Introduction}

Brain tumors including meningioma are seen in patients with Parkinsonism and Parkinson's disease (PD). ${ }^{1}$ Certain identified genetic abnormalities associated with meningioma and other brain tumors suggest potential genetic linkage with PD as well.2,3 We document a case of concurrent Parkinsonism and meningioma that manifested in a woman with acute quadriplegia and decreased responsiveness where surgery was successful despite the presence of cerebellar tonsillar herniation.

\section{Case Report}

A 56-year-old Afro-Trinidadian woman was brought to the accident and emergency department of hospital with acute onset of paralysis which began in both lower limbs and spread to involve the both upper limbs in a period of 3 days and was associated with decreased responsiveness for 1 day. She was attending the neurology outpatient clinic at another hospital where based on her twoyear history of uncomplicated stage II (Hoehn and Yahr) Parkinson's disease (PD) characterized by bradykinesia, rigidity, bilateral upper tremor more prominent in the right side, and cogwheel rigidity at the wrists, she was eventually diagnosed as having a Parkinson-plus syndrome with poor response to carbidopa-levodopa (25-100 mg) orally three times daily and trihexyphenidyl HCL 2 $\mathrm{mg}$ orally three times daily.

There was also a history of paroxysmal vertigo, tinnitus, mild subjective hearing loss, and worsening gait disturbances for 6 months. Brain imaging or other investigations to clarify this vestibulocerebellar syndrome had not been done previously. She had no children, personal history of other medical disease or surgical procedures, or exposure to chemicals, ionizing radiation, recreational drugs, alcohol or smoking. There was no family history of neoplasm or neurological diseases.

On initial examination she was afebrile $\left(37.3^{\circ} \mathrm{C}\right)$, anicteric, tachycardic (110 beats per minute) and normotensive (140/70 mm Hg). Her oxygen saturation was well maintained on room air $(100 \%)$ and she was not tachypneic (20 breath per minute). Patient's body mass index (BMI) was 32 units per $\mathrm{kg} / \mathrm{m}^{2}$. Examination revealed a Glasgow Coma Scale of 10/15 with disorientation to time and place but not to person. Parkinson's facies, right VI nerve palsy and global weakness were observed. Power score in all limbs was 0/5 according to the Medical Research Council scale which compromised the undertaking of diadochokinesis and finger-nose-finger tests. Hyperreflexia was present in all limbs. Babinski's sign was
Correspondence: Antonio Jose Reyes, Neurology Unit, San Fernando Teaching Hospital, Independence Avenue, San Fernando, Trinidad, West Indies.

Tel.: +18687221270 .

E-mail: reyesax@yahoo.com

Key words: posterior fossa mass, meningioma, parkinsonism, Parkinson disease, quadriplegia.

Acknowledgements: We thank Miss Sharon Sealy for preparation of the images. We thank the patient for allowing us to report her clinical data.

Contributions: The idea for reporting this case was that of AJR and KR. AJR drafted the first draft of the manuscript. Further intellectual content and editing was done by AJR, KR, MA, WG and FR. FR also provided advice on selection and interpretation of the radiological images. MA performed the tumour surgical removal. WG prepare the histopathology aspect of the case. All authors saw, edited and approved the final contents. KR assumes responsibility for the integrity of the contents.

Conflict of interest: the authors have no potential areas of conflict to declare.

Funding: none.

Received for publication: 8 August 2018 . Revision received: 16 September 2018.

Accepted for publication: 2 January 2019.

This work is licensed under a Creative Commons Attribution NonCommercial 4.0 License (CC BY-NC 4.0).

(C) Copyright A.J. Reyes et al., 2019

Licensee PAGEPress, Italy

Neurology International 2019; 11:7815

doi:10.4081/ni.2019.7815

negative bilaterally with horizontal gaze nystagmus positive to the left. Neck rigidity was found with Kernig and Brudziński signs negative bilaterally. Other cranial nerves and sensory system were intact. The rest of the physical examination was normal.

Extensive investigations for infectious, autoimmune, and metastatic diseases were negative. Cerebral Magnetic Resonance Imaging (MRI) showed a solid, well circumscribed T2 isointense, left posterior cranial fossa extra-axial mass, measuring $3.7 \times 3.5 \times 4.0 \mathrm{~cm}$, arising from the left cerebellopontine angle, causing cerebellar tonsillar herniation of $6 \mathrm{~mm}$ beyond the foramen magnum, best seen in a midline sagittal $\mathrm{T} 1$ weighted view (Figure 1A). This compresses the 
left cerebellar hemisphere and brainstem, best seen in T2 weighted coronal and T2 weighted axial views (Figure 1B,C), and midbrain distortion seen in $\mathrm{T} 1$ weighted and fluid-attenuation inversion recovery weighted axial views (Figure 1D,E). The mass was homogeneous in appearance, likely benign and enhanced homogeneously upon administration of intravenous Gadolinium. Magnetic Resonance Angiography scan (cerebral MRA scan) was normal. PET CT scan was unavailable.

The tumor was surgically removed, histology and immunohistochemistry were consistent with a meningioma (Figure 2).

There was a slow progressive good postoperative recovery within 14 days of ICU/High Dependency Unit care. Mechanical ventilatory support was given for 10 days. She received 7 days intravenous (IV) Vancomycin $1 \mathrm{~g}$ three times daily for a Hospital-Acquired Pneumonia with a positive methicillin resistant coagulase negative staphylococcus growth from tracheostomy secretion sampled. Hospital-Acquired Pneumonia was satisfactory resolved. After that she was gradually alert and oriented. She was discharged 21 days after admission on carbidopa-levodopa (50-200 mg) orally three times daily for outpatient clinic follow up. Figure 3A, a T2 weighted MRI axial view showed post-surgical tumor cavity with edema and an extra axial fluid collection posterior to the cerebellum. The patient's hand writing at admission was illegible (Figure 4A) but it improved 6 weeks after surgical intervention (Figure 4B). Figure 3B, a T1 weighted MRI axial view of the brain showed post-surgical tumor cavity 3 months later, with resolution of the extra-axial collection. Although, parkinsonism persists despite tumor removal and postsurgical treatment, the patient improved, walking without support 3 years later. She remains oriented to time, person, and place with a Glasgow Coma Scale of 15/15. Currently, 8 years later, she is at stage II (Hoen and Yahr), with persistent tremor but no vertigo, tinnitus, hearing loss, VI nerve palsy, nystagmus or significant gait disturbances. However, most differential diagnosis was ruled out (Table 1) with no evidence of autonomic neuropathy or features of progressive supranuclear palsy, multisystem atrophy or corticobasal degeneration.

\section{Discussion}

Parkinson's disease (PD) is a progressive and chronic non-communicable degenerative disorder of the central nervous system clinically characterized by four main types of movement disorders: a) Tremor: Trembling in the hands, arms, legs, jaw and/or head; b) Rigidity: Stiffness of the limbs and trunk; c) Bradykinesia: Slowness of movement; and d) Postural instability: impaired balance. These symptoms usually begin gradually and worsen with time. As they become more pronounced, patients may have difficulty walking, talking, or completing other simple tasks. ${ }^{1-5}$ There are a variety of different causes of parkinsonism including PD, secondary parkinsonism, and the parkinsonism plus syndromes. Secondary parkinsonism is caused by structural, toxic, metabolic, or infectious mechanisms. Among structural causes, intracranial neoplasms are a rare cause of secondary parkinsonism. 1,2,6

In recent years, increasing amount of epidemiological evidence points to a positive association between PD and cancers through several common genetic mutations in SNCA, PARK2, PARK8, ATM, $\mathrm{p} 53$, PTEN, and MC1R resulting in cellular changes such as mitochondrial dysfunction, aberrant protein aggregation, and cell cycle dysregulation. Genes multiple mutations associated to PD and neoplasm implies environmental and occupational factors involved. Although some cases of PD are hereditary and can be traced to specific genetic mutations, most cases are sporadic. PD and parkinsonism likely result from a combination of genetic susceptibility and exposure to one or more environmental factors that trigger the diseases. PD and neoplasm geneticenvironmental links are poorly understood globally and cost-efficient therapeutic approaches for these cases remain unexplored. $1,2,4,5,7$

Epidemiological data support the increased risk of melanoma or breast cancer in patients with PD.1,6 On the other hand, the methylazoxymethanol metabolite formaldehyde is linked to the risk for $\mathrm{PD}$, prostate cancer, and skin cancer, including melanoma.6,8-11 Depending on location, histology and extension of infiltration, any brain tumor has the potential to cause Parkisonism. In brain neoplasm the cells reproduce out of control and fail to die but in PD the neurons that release the neurotransmitter dopamine in the substantia nigra region of the brain die. The main risks factors for brain tumors and/or
Parkinsonism are shown in Table 1.1-11 Reasons for these associations remain unknown.

Some cases of Parkinsonism may be associated with arterial compression of the cerebral peduncle by the posterior cerebral artery which may be seen on MRI studies. Improvement and prolonged relief of $\mathrm{PD}$ and/or parkinsonism in those patients may be possible in certain circumstances by the use of microvascular decompression of the brainstem. ${ }^{12}$

Meningioma is the second most common intracranial tumor and has been shown to demonstrate heterogeneous clinical features according to tumor size and location. Most meningiomas are slowly growing and result in benign clinical outcomes, but only $20 \%$ exhibit clinically aggressive features.

Although it is rare for intracranial space-occupying lesions to present with a Parkinsonism, a few cases of parkinsonism due to meningioma have been reported.1-11 Parkinsonism has been associated with space occupying lesions in the frontal lobe, temporal lobe, parietal lobe, septum pellucidum, brainstem, parasagittal region, suprasellar and sphenoidal wing regions. Kaijima et al., reviewed 58 cases of Parkinsonism associated with intracranial space occupying lesions, out of which 8 were infiltrating gliomas involving thalamus, basal ganglia or midbrain. ${ }^{13}$ Four supratentorial tumor sites are described in literature as being located in the frontal lobe, temporal lobe, parietal lobe and thalamus. ${ }^{14,15}$ An extensive review showed that almost all reported extrinsic occupying space lesions of the brain have been meningiomas in these patients. Gliomas and metastatic tumors in the frontal lobes have also been associated with Parkinsonian tremor. Subdural hematomas have also been reported producing Parkinsonism. ${ }^{16}$ Midline extrinsic lesions of the brain producing movement disorders are even more rare. Among them are anterior third ventricular meningioma, pituitary adenoma and craniopharyngioma. ${ }^{16}$ Rarely parkinsonism may be seen due to posterior fossa lesions. ${ }^{16-20}$ Nearly all symptoms are possibly produced by compression of basal ganglia. ${ }^{12-}$ 20 Quadriplegia and possibly Parkinsonism in our patient could have occurred secondary to peritumoral edema, compression due to mass effect, raised intracranial pressure, transient tumoral attack, blood flow changes induced by the mass effect, or a combination of some of these factors. The most likely cause of the quadriplegia in our patient was due to cerebellar tonsillar herniation into the 


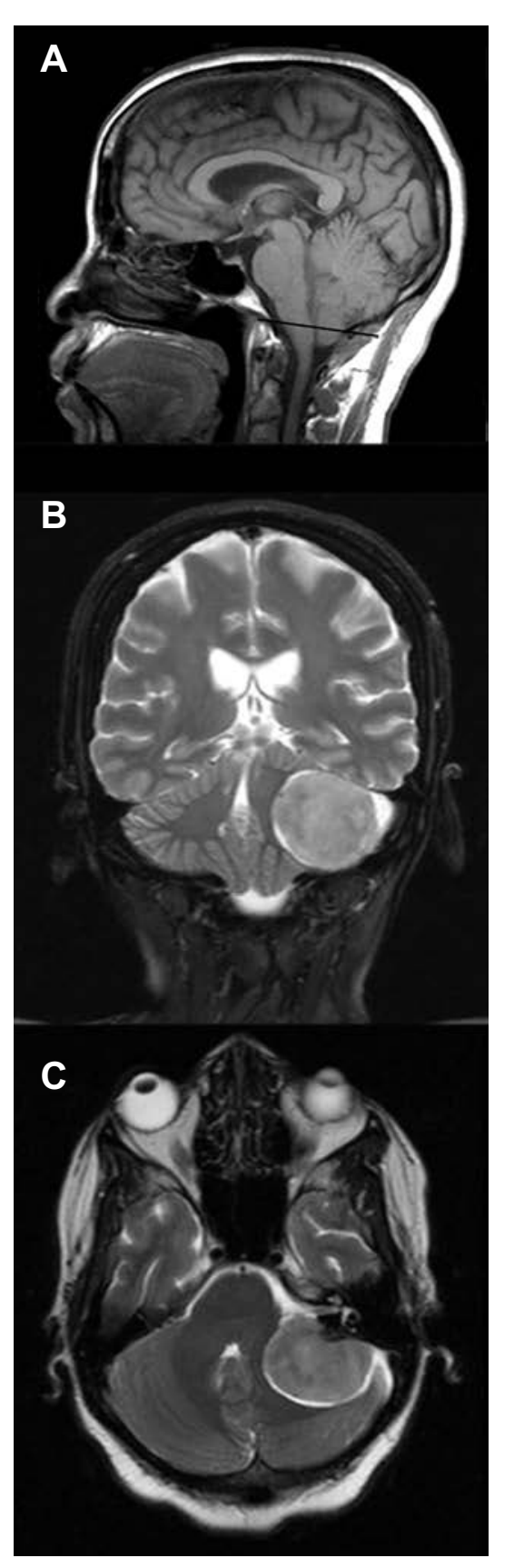

upper cervical canal from increased intracranial pressure, edema and/or mass effect. The cerebellar tonsil involved moved downward through the foramen magnum possibly causing compression of the ventral region of the lower brainstem and upper cervical spinal cord as they pass through the foramen magnum (Figure 1). This is a life-threatening condition as it causes increased pressure on the medulla oblongata which contains respiratory and cardiac control centers. Cases of limb weakness due to meningioma in the vicinity of the foramen magnum, or located in the clivus-anterior to the medulla, or in the left frontoparietal region of the brain, have been reported. ${ }^{20}$ We believe that a combination of cerebellar tonsillar herniation, and compression of brainstem and midbrain caused most of the acute and subacute symptoms and signs in our patient. Complete rapid reversibility of those clinical manifestation after removal of the tumor without recurrence of limb weakness or decreased responsiveness 8 years after surgery are also in keeping with that hypothesis. Furthermore, the Parkinsonism has not progressed.

Efficient treatment involved several disciplines spanning the medicine-surgery spectrum and both secondary and tertiary

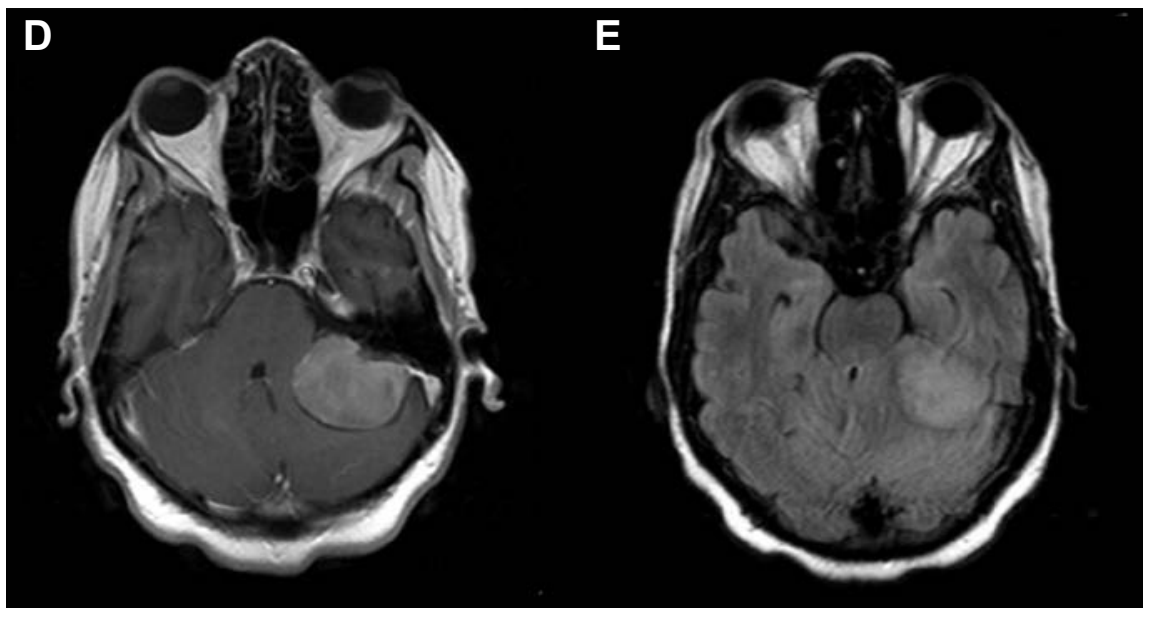

Figure 1. A) Middle Sagittal T1 weighted MRI image showing cerebellar tonsillar herniation of $6 \mathrm{~mm}$, determined by extension beyond a reference line from the bastion to opisthion. B) T2 weighted MRI coronal view of the brain demonstrating a solid T2 isointense left cerebellopontine angle mass, measuring $3.7 \times 3.5 \times 4.0 \mathrm{~cm}$, with mild compression of the brainstem. C) T2 weighted MRI axial view showing the posterior fossa tumor in the left cerebellopontine angle compressing cerebellum. D) T1 weighted axial post intravenous Gadolinium administration demonstrating homogeneous enhancement of the left cerebellopontine angle mass, which is a typical feature of a meningioma. There is no extension to the left internal auditory meatus to suggest an acoustic neuroma. E) Fluid-attenuated inversion recovery MRI axial view of the brain showing left posterior fossa tumor with mild distorted midbrain.

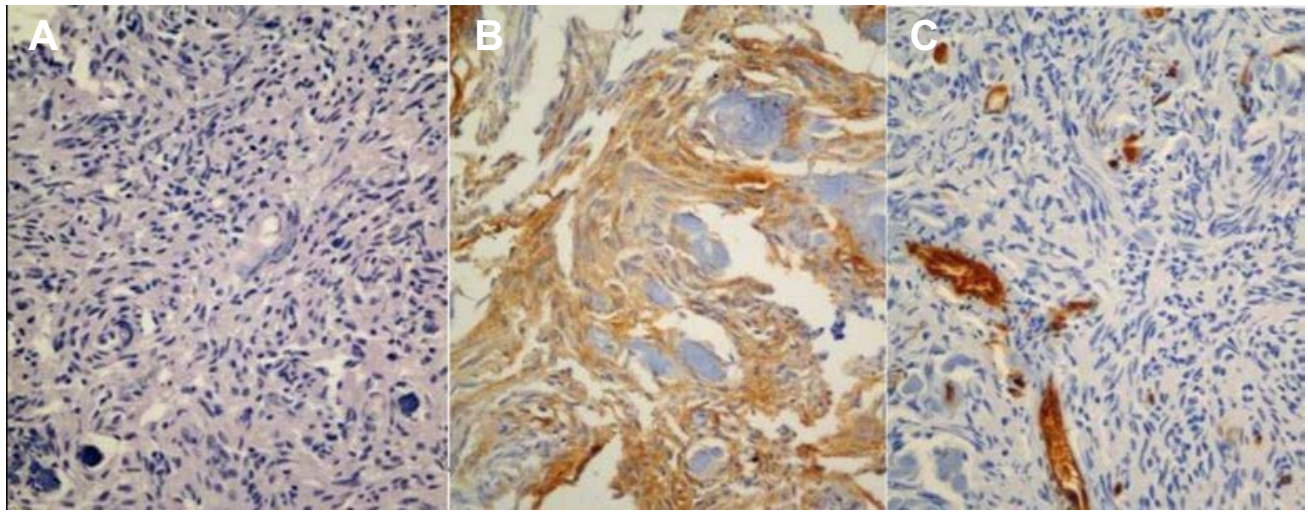

Figure 2. A) Photomicrography (Magnification 100x) of the posterior fossa tumor with routine H\&E staining showing a spindle cell tumor with a somewhat whorled growth pattern typical of meningothelial meningioma. B) The tumor cells were positive for Epithelial Membrane Antigen expression by immunohistochemistry. C) Tumor cells were negative for CD34 and S-100, thus ruling out solitary fibrous tumor and schwannoma respectively. 
care. Potential surgical reversibility of the neurological manifestations associated to the brain tumor, poses an imperative for early diagnosis and management. PD is the most common form of Parkinsonism, the name for a group of disorders with similar features and symptoms. No more than one in 1,000 diagnoses of PD are in reality parkinsonism due to an unrecognized neoplasm, especially prostate cancer, melanoma and brain tumors. 1,2,4,5,6,18-20 Neuronal loss in the substantia nigra, compression to the basal ganglia, distortion to the midbrain and basal ganglia, brain network disturbances between the basal ganglia and supplementary motor area, and old age have been implicated. ${ }^{1}$ Our patient clinical presentation with acute onset quadriplegia, decreased responsiveness, and a previous short history of PD stage II (Hoehn and Yahr) with bilateral parkinsonism affecting more the right side of the body with poor response to carbidopa-levodopa and rapid neurological deterioration, should have suggested the presence of a space occupying lesion of the brain. Global hyperreflexia with horizontal gaze nystagmus to the left and right VI nerve palsy should have also prompted a brain scan imaging study. Although patient clinical deterioration could have been wrongly attributed to progressive
$\mathrm{PD}$, the previous history of paroxysmal vertigo, tinnitus, mild subjective hearing loss, and worsening gait disturbances for 6 months, were in keeping with a cerebello- pontine angle mass or schwannoma. Under this circumstance, the brain scan imaging study could have been done 6 months previously to the acute onset quadriplegia and
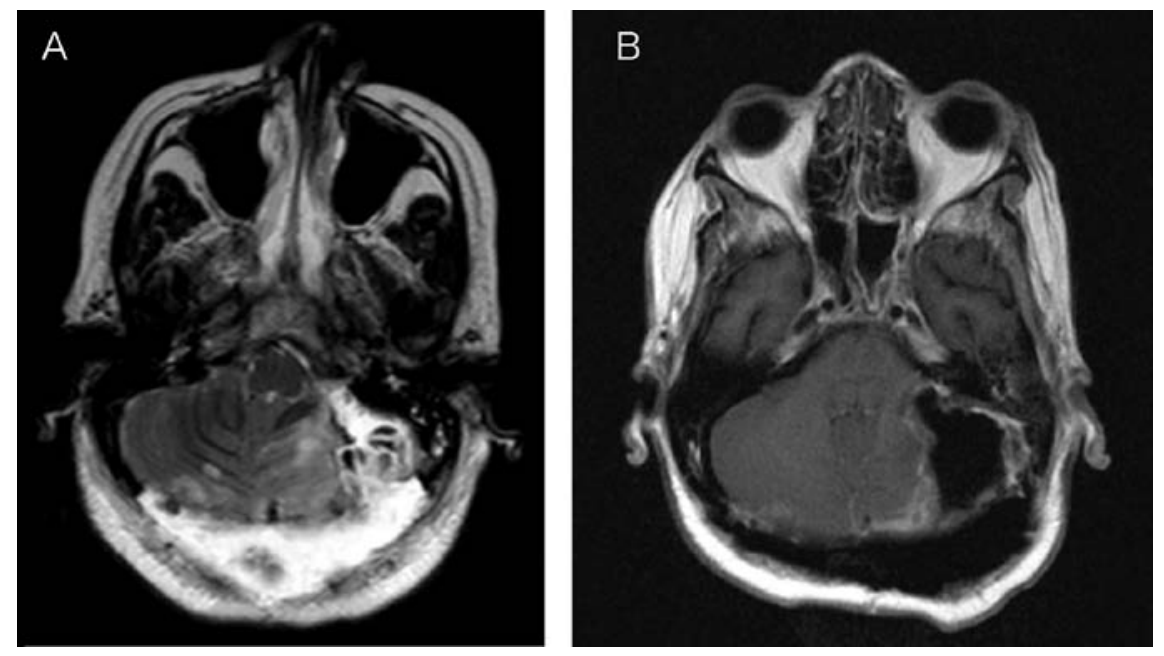

Figure 3. A) T2 weighted MRI axial view of the brain showing post-surgical tumor cavity, with edema and an extra axial fluid collection posterior to the cerebellum. B) T1 weighted MRI axial view, post intravenous Gadolinium, showing post-surgical tumor cavity, without edema. Post-surgical enhancement is seen in the tumor bed. No residual or extra axial fluid collection was seen.

Table 1. Main risks factors for brain tumors and/or parkinsonism.

\begin{tabular}{|c|c|c|}
\hline Risk Factor & Tumors & Common Causes \\
\hline Exposure to ionizing radiation & Meningiomas, sarcomas, and malignant gliomas & $\begin{array}{l}\text { DNA mutations: Substitutions and frameshift } \\
\text { mutations at any level }\end{array}$ \\
\hline Von Recklinghausen disease & Schwannomas, gliomas, meningiomas, neurofibromas & Changes in the NF1 gene \\
\hline Neurofibromatosis type 2 & Bilateral or unilateral vestibular schwannomas, meningiomas & Changes in the NF2 gene \\
\hline Von Hippel-Lindau disease & $\begin{array}{l}\text { Hemangioblastomas of the Central Nervous System } \\
\text { (CNS), eye, ear, kidney, adrenal gland, and pancreas }\end{array}$ & Changes in the $V H L$ gene \\
\hline Li-Fraumeni syndrome & $\begin{array}{l}\text { Gliomas, breast cancer, sarcomas, leukemia, adrenal } \\
\text { gland cancer, among other types of cancer }\end{array}$ & Changes in the TP53 gene \\
\hline Tuberous sclerosis & $\begin{array}{l}\text { Astrocytoma, benign tumors of the brain, skin, heart, , } \\
\text { kidneys and other organs }\end{array}$ & Changes in either the $\mathrm{TSCl}$ or the $\mathrm{TSC} 2$ gene \\
\hline $\begin{array}{l}\text { Gorlin syndrome, Turcot syndrome } \\
\text { and Cowden syndrome }\end{array}$ & CNS Tumors & $\begin{array}{l}\text { Changes in genes: PTCH, APC, MLH1, PMS2, } \\
\text { PTEN, SDHB, } \\
\text { SDHD, and KLLN }\end{array}$ \\
\hline Impaired immune system & Lymphomas of the brain & Acquired immunodeficiency syndrome (AIDS) \\
\hline Drugs & No tumors but parkinsonism & $\begin{array}{l}\text { Phenothiazine, chlorpromazine, prochlorperazine, } \\
\text { perphenazine, fluphenazine, promethazine, } \\
\text { butyrophenones, haloperidol, diphenylbutylpiperidine, } \\
\text { pimozide, benzamide substitute, sulpiride, } \\
\text { risperidone, olanzapine, ziprasidone, aripiprazole, } \\
\text { reserpina, tetrabenazine, metoclopramide, } \\
\text { levosulpiride, clebopride, flunarizine, cinnarizine, } \\
\text { lithium, clozapine, quetiapine, valproic acid, } \\
\text { phenitoine, domperidone, itopride, fluoxetine, } \\
\text { sertraline, paroxetine and citalopram }\end{array}$ \\
\hline Suspected but unproven risk factors & CNS tumors & $\begin{array}{l}\text { Organic solvents and other hydrocarbons, zinc, } \\
\text { paraquat, rotenone, maneb, manganese, } \\
\text { MPTP (1-methyl 4-phenyl 1,2,3,6-tetrahydropyridine), } \\
\text { toluene, N-hexane, carbon disulfide, carbon monoxide, } \\
\text { mercury, cyanide, copper, lead, trichloroethylene, } \\
\text { household paint, varnishes and garden sprays, cell } \\
\text { phones, vinyl chloride and aspartame }\end{array}$ \\
\hline
\end{tabular}




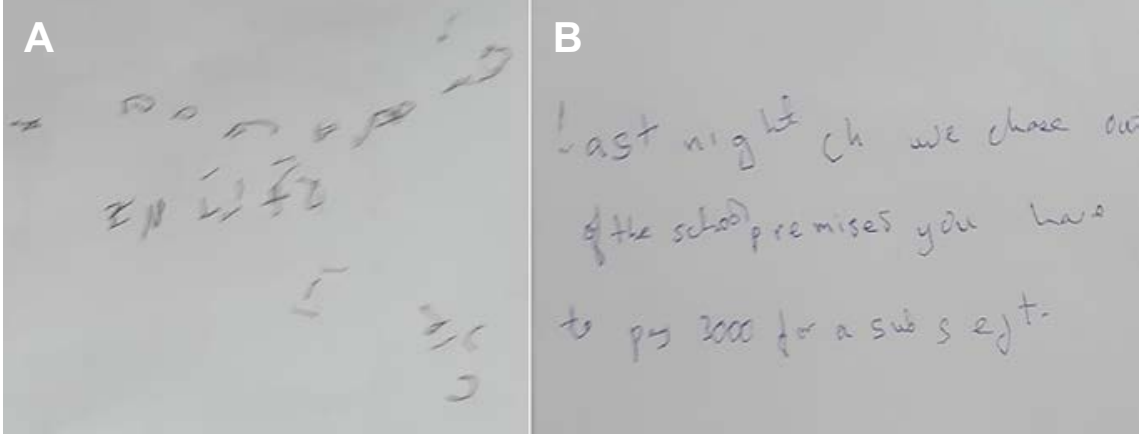

Figure 4. Patient hand writing illegible before (A) and six weeks after surgery (B) showing improvement.

decreased responsiveness when the tumor was smaller but detectable. MRI scan images showing a large left cerebellopontine angle tumor with brainstem and midbrain distortion, compression of the cerebellum with cerebellar tonsillar herniation were in keeping with the clinical manifestations in our patient. T1 weighted axial post IV Gadolinium administration demonstrated homogeneous enhancement of the left cerebello-pontine angle mass, which is a typical feature of a meningioma. There was no extension to the left internal auditory meatus to suggest an acoustic neuroma (Figure 1D). Previously responsive or unresponsive PD patients with unexpected deterioration despite conventional treatment should prompt revaluation for new underlying disease preventing late diagnosis.

\section{Conclusions}

In conclusion, we report a rare case of a large left posterior fossa meningioma in an Afro-Trinidadian woman with carbidopa-levodopa resistant parkinsonism who presented with acute onset quadriplegia and decreased responsiveness. We highlight the surgical removal of the tumor that led to complete permanent reversibility of the neurological manifestations associated to the cerebellar tonsillar herniation into the foramen magnum, mass effect on the brainstem, midbrain region and cerebellum previously correlated by clinical findings and MRI studies. This case reminds clinicians that Parkinsonian-like patients with dopa unresponsiveness and/or acute neurological deficit or deterioration should be worked up for other possible causes.

\section{References}

1. Yasuhara T, Agari T, Kambara H, et al. Parkinsonism Related to Brain the Literature. Open Neurosurg J 2009;2:4-7.

2. Garber K. Parkinson's disease and cancer: the unexplored connection. JNCI J Natl Cancer Inst 2010; 102:371-4.

3. Lin PY, Chang SN, Hsiao TH, et al. Association Between Parkinson Disease and Risk of Cancer in Taiwan. JAMA Oncol 2015;1:633-40.

4. Freedman M, Wu J, Chen $\mathrm{H}$, et al. Associations between cancer and Parkinson's disease in U.S. elderly adults. Int J Epidemiol 2016;45:741-51.

5. Kisby GE, Spencer PS. Parkinsonism and Cancer. JAMA Neurol 2013;70:414-5.

6. Ye R, Shen T, Jiang Y, et al. The Relationship between Parkinson Disease and Brain Tumor: A MetaAnalysis. PLoS One 2016;11: e0164388.

7. Feng DD, Cai W, Chen X. The associations between Parkinson's disease and cancer: the plot thickens. Translat Neurodegener 2015;4:1-14.

8. Springborg JB, Poulsgaard L, Thomsen J. Nonvestibular Schwannoma Tumors in the Cerebellopontine Angle: A Structured Approach and Management Guidelines. Skull Base 2008;18:21727

9. Wartenberg D, Reyner D, Scott CS. Trichloroethylene and cancer: epidemiologic evidence. Environ Health Tumors: A Case Report and Review of
Perspect 2000;108:161-76

10. Gendron C. Cancers à Shannon: une histoire d'eau Enquète. RadioCanada.ca. Available from http://www.radio-canada.ca/emis$\mathrm{s}$ i o n s/e n que te / 2008 2009/reportages.asp.

11. Public Health Assessment Agency for Toxic Substances and Disease Registry. Potential Impact of TCE Exposure, Hidden Lane Landfill Sterling, Loudoun County, Virginia 2009. Available from: http://www.atsdr.cdc.gov/HAC/pha/Hid denLaneLandfill/HiddenLaneLandfillP ublicH ealthAssessment (PublicComment)1-12-09.pdf.

12. Jannetta PJ, Whiting DM, Fletcher LH, et al. Parkinson's disease: an inquiry into the etiology and treatment. Neurol Intern 2011;3:e7.

13. Kaijima M, Fukui H, Shima F, et al. Epidermoid in the middle cranial fossa presenting hemiparkinsonism. A case report. Neurol Surg 1978;6:11038.

14. Polyzoidis KS, McQueen JD, Rajput $\mathrm{AH}$, MacFadyen DJ. Parkinsonism as a manifestation of brain tumour. Surg Neurol 1985;23:59-63.

15. Saleem SM, Kirmani A, Dhobi GN. An Unusual Case of Parkinsonism secondary to right parasagittal meningioma. Neurol India 2000;48:190.

16. Bhatoe HS. Movement disorders caused by brain tumours. Neurol India 1999;47:40

17. Farlow J, Pankratz ND, Wojcieszek J, Foroud T. Parkinson Disease Overview 2004 May 25. In: Adam MP, Ardinger HH, Pagon RA, et al., eds. GeneReviews ${ }^{\circledR}$ [Internet]. Seattle (WA): University of Washington, Seattle 1993-2018. Available at https://www.ncbi.nlm.nih.gov/books/N BK1223/

18. de Vera Royes JA. Parkinsonism-like syndrome caused by posterior fossa tumour. J Neurosurg 1970;33:599601.

19. Oliver L. Parkinsonism due to midbrain compression. Lancet 1982;11: 817-9.

20. Poppen JL, Skwarok EW. Left Frontoparietal Meningioma with Quadriplegia. J Neurosurg 1953;10: 182-4. 